\title{
O lugar da farmacognosia na formação em farmácia: questões epistemológicas e suas implicações para o ensino
}

\author{
Wellington Barros da Silva* \\ Programa de Pós-graduação em Educação Científica e Tecnológica, Centro de Ciências Físicas e Matemáticas, \\ Universidade Federal de Santa Catarina, 88040-900 Florianópolis-SC, Brasil \\ Curso de Farmácia, Universidade Federal de Sergipe, UFS, Laboratório de Ensino e Pesquisa em Farmácia Social \\ (LEPFS), Cidade Universitária Prof. "José Aloisio de Campos", Av. Marechal Rondon, s/n Jardim Rosa Elza, \\ 49.100-000 São Cristovão-SE, Brasil.
}

\begin{abstract}
RESUMO: O presente ensaio propõe a discussão de questões epistemológicas referentes ao ensino de Farmacognosia e seu papel na formação e apropriação de uma cultura científica entre os estudantes dos cursos de Farmácia, além das possibilidades de contribuição da disciplina para o estabelecimento de um novo modelo de prática profissional. Defende que há uma estreita relação entre a crise de identidade profissional da Farmácia e incongruências epistemológicas na matriz disciplinar da Farmacognosia, o que, do ponto de vista pedagógico implica em obstáculos para o processo de ensino e aprendizagem da disciplina. Além disso, o caráter experimental da Farmacognosia comporta uma concepção de ciência e do objeto de conhecimento que influenciam na seleção de conteúdos e pode constituir obstáculos na mediação e apropriação de conhecimentos e demandas necessárias para a prática profissional da Farmácia.
\end{abstract}

Unitermos: Ensino farmacêutico, farmacognosia, epistemologia.

\begin{abstract}
The place of pharmacognosy in the pharmaceutical education: epistemological issues and their implications for teaching." This paper proposes the discussion of epistemological issues concerning the teaching of pharmacognosy and its role in training and ownership of a scientific culture among students of the courses of Pharmacy, in addition to the potential contribution of the discipline for the establishment of a new model of professional practice. It argues that there is a close link between the crisis of professional identity of Pharmacy and epistemological inconsistencies in the disciplinary matrix of pharmacognosy, which from a teaching point of view it involves obstacles to the process of teaching and learning the discipline. Moreover, the experimental nature of pharmacognosy includes a conception of science and the object of knowledge that influences the selection of content and may constitute obstacles in mediation and appropriation of knowledge and demands for the pharmacy practice.
\end{abstract}

Keywords: Pharmaceutical education, pharmacognosy, epistemology.

\section{INTRODUÇÃO}

Uma abordagem inicial voltada para a discussão sobre o ensino da Farmacognosia e seu papel nos currículos de Farmácia deve começar pela formulação de algumas questões necessárias à problematização do tema, de tal forma que ajude a elucidar sua instrumentação para a formação de farmacêuticos. No Brasil, por iniciativa da Sociedade Brasileira de Farmacognosia, esta questão vem sendo debatida de forma recorrente nos encontros e simpósios de natureza científica.

Talvez a comunidade científica da área, envolvida diretamente com a produção de conhecimento, mas também com o ensino de graduação daquela disciplina, considere este assunto, em alguma medida, já superado. Todavia, uma rápida revisão sobre o estado da arte da produção acadêmica referente ao ensino de Farmacognosia, ou à inserção da fitoterapia nos currículos de farmácia, reforça a hipótese de que esta discussão precisa articular de forma equilibrada as demandas inerentes ao avanço da pesquisa e desenvolvimento (P\&D) em produtos naturais, com sua integração nas atividades de provisão de cuidado, bem como na promoção do uso destes recursos terapêuticos (Shah et al., 2005; Dutta et al., 2003; Shields, 2003; Mackowiak et al., 2001).

Como pretendemos argumentar neste artigo há pelo menos três aspectos a partir dos quais a Farmacognosia assume relevância para a formação profissional e científica do farmacêutico: a) o significado que a apropriação do conhecimento relativo aos produtos biologicamente ativos de origem natural pode ter para o exercício da prática farmacêutica; b) a contribuição do ensino da 
Farmacognosia no desenvolvimento de uma cultura científica e; c) os aspectos epistemológicos implícitos na organização pedagógica e suas implicações no ensino da disciplina.

\section{CONTRIBUIÇÕES DA FARMACOGNOSIA PARAA MISSÃO DA PRÁTICA FARMACÊUTICA}

Tomando como ponto de partida dois artigos interessantes publicados por Tyler (1996) e Chang e colaboradores (2000), que argumentaram respectivamente sobre a relevância e necessidade do farmacêutico dominar um conjunto de conhecimentos referentes à indicação e uso de plantas medicinais, ao mesmo tempo em que identificaram deficiências na aplicação destes conhecimentos; estabelecemos uma das questões centrais deste ensaio: qual a efetiva contribuição da farmacognosia para a missão da prática farmacêutica? Pergunta que, para ser adequadamente respondida remete a outra questão: qual a missão ou o que a sociedade espera da prática farmacêutica? Desta reflexão deriva um conjunto de atribuições específicas característicos do modelo de prática, que representam a aplicação de conhecimentos, habilidades e atitudes constituintes do rol de competências de um farmacêutico, cujo exercício profissional esteja orientado para a consecução da sua missão ou objeto social.

Segundo o documento elaborado pela Federação Farmacêutica Internacional (FIP), sob os auspícios da Organização Mundial da Saúde (OMS), denominado Boas Práticas de Farmácia, "a missão da prática farmacêutica consiste em administrar medicamentos e outros produtos e serviços para o cuidado da saúde, e ajudar as pessoas e a sociedade a utilizá-los da melhor maneira possível" (IPF, 1997). Isto significa dizer que o processo de uso de medicamentos e outros recursos terapêuticos configuram o objeto social da Farmácia. Neste sentido, considerando o contexto brasileiro, onde há um forte apelo mercadológico de indução do consumo de "produtos naturais", associado ao senso popular que atribui prestígio e significativo valor cultural no uso de plantas medicinais, a aceitação daquela missão por si justifica a pertinência do ensino da Farmacognosia e disciplinas afins na formação de futuros farmacêuticos em nosso país.

Outrossim há de se ressaltar a recente elaboração de políticas públicas voltadas para a área, como o Decreto No. 5.813 (2006) que institui a Política Nacional de Plantas Medicinais e Fitoterápicos. Espera-se deste modo que o farmacêutico seja capaz de promover o uso racional de plantas medicinais e produtos fitoterápicos na perspectiva da integralidade das ações e da complementaridade de modelos e práticas de promoção da saúde, que considerem ao mesmo tempo saberes e práticas das medicinas tradicionais, o legado cultural inerente ao contexto histórico do uso de espécies vegetais, as exigências éticas e a rigorosidade científica do processo de cuidado à saúde.
Além disso, face ao cenário econômico e geopolítico deste início de século há um desafio particular para o ensino de Farmacognosia no contexto brasileiro: articular a complexidade social e política, que faz parte da questão do aproveitamento dos recursos naturais e da biodiversidade, ao conteúdo da disciplina. Tal opção pedagógica corresponde a uma necessidade estratégica para a formação crítica de recursos humanos nesta área, mas também de preservação do patrimônio de saberes e práticas de comunidades tradicionais, um compromisso ético historicamente esquecido nas estratégias e ações de PD \& I da área.

Com este enfoque na disciplina, um farmacêutico com formação científica crítica em Farmacognosia, seja como pesquisador ou como um profissional de saúde, poderá disponibilizar informação, produtos e práticas de promoção à saúde validadas a partir dos recursos da sociobiodiversidade presente nos diferentes biomas do nosso país.

Se com estes argumentos iniciais parece óbvia a importância de, no escopo da disciplina, abordar os aspectos referentes à prospecção, processamento, promoção e preservação de recursos naturais de interesse biológico e, mais ainda; se há consenso entre os professores da área que cabe a ela dar conta desta tarefa; então por que uma discussão sobre o papel da Farmacognosia nos currículos de formação de farmacêuticos?

Necessariamente uma possibilidade de resposta remete aos mecanismos de institucionalização, de controle e regulação profissional, já que do ponto de vista histórico a Farmacognosia delimitou e configurou até certo ponto o processo de construção da Farmácia como ofício, como profissão e como ciência.

Para entender a sua função na formação dos estudantes é preciso avaliar a posição da disciplina na consolidação das Ciências Farmacêuticas como locus de desenvolvimento científico, do alvorecer da revolução científica até o período da revolução farmacológica já na sociedade industrializada; bem como o significado que a área representou no fenômeno de profissionalizaçãodesprofissionalização-reprofissionalização da Farmácia, principalmente a partir do processo de industrialização da produção de medicamentos e com o advento do chamado movimento clínico da Farmácia nos Estados Unidos por volta da década de 1960. Analisada sob este prisma, a Farmacognosia experimentou uma dinâmica de expansão e desenvolvimento científico ao mesmo tempo que uma diminuição do status e do prestígio da disciplina, inclusive com pressões para a redução de carga horária ou da sua supressão nas matrizes curriculares dos cursos de graduação.

A percepção aqui levantada apenas como uma hipótese que mereceria maior elucidação, considera que, em um universo onde proliferam no país faculdades e cursos de farmácia com grande heterogeneidade de propostas curriculares e de qualificação docente, há um 
descompasso entre o que se pesquisa e o que se ensina sobre Farmacognosia, o que de certa forma refletiria de um lado a crise de identidade profissional que comporta incongruências epistemológicas que talvez merecessem a atenção da comunidade científica.

Considerando a validade da argumentação apresentada até aqui no texto, há duas possibilidades de resposta imediata para o problema do protagonismo da Farmacognosia nos cursos de graduação: a função da Farmacognosia poderia ser de um lado contribuir na formação, instrumentalizando o futuro farmacêutico para o exercício e cumprimento da sua missão profissional; mas também por outro lado, ela certamente contribui (concordaríamos quase todos) para o desenvolvimento de competências cognitivas, de habilidades de técnicas experimentais que introduziriam o aluno na abordagem científica da investigação de substâncias bioativas promissoras, da caracterização e padronização de formas extrativas, do fracionamento e isolamento de compostos; enfim, a Farmacognosia tem um papel como disciplina básica na iniciação dos alunos dos Cursos de Farmácia naquilo que poderia ser designado como matriz disciplinar ou paradigma das Ciências Farmacêuticas (Kuhn, 2005).

Considerando o estatuto histórico daquela disciplina, primeiro como área que configurou durante séculos a arte e o ofício da Farmácia e logo em seguida, como disciplina constituinte da base científica das ciências farmacêuticas, estas possibilidades apresentam desdobramentos que trazem consigo implicações epistemológicas e pedagógicas, com conseqüências para a formação dos alunos não só como farmacêuticos ou cientistas, mas também como cidadãos em um mundo cada vez mais permeado pela ciência e pela tecnologia.

Olhando para o passado, é certo que a arte de curar, ou dito de outro modo, a utilização de recursos naturais para o tratamento de enfermidades, acompanha o homem desde tempos remotos. O domínio das técnicas de preparo de remédios e poções representa, junto com tantas outras habilidades desenvolvidas pelo ser humano, o resultado da sua capacidade em estabelecer relações e representações de si mesmo e do meio, de mobilizar recursos que viabilizem sua necessidade de adaptação e ao mesmo tempo uma resposta ao desafio civilizatório.

\section{O PROBLEMA EPISTEMOLÓGICO FARMACOGNOSIA}

DA

A epistemologia é o ramo da filosofia que se ocupa do questionamento acerca da natureza, origem e da validade do conhecimento, especificamente de um tipo de conhecimento elaborado que designamos como conhecimento científico. Entre as questões ou problemas que ela busca responder encontra-se a definição sobre o que é o conhecimento e como nós o alcançamos, ou seja, qual a natureza da relação entre o indivíduo que conhece e o objeto do conhecimento.
No domínio das ciências farmacêuticas a (re) definição do objeto do conhecimento da Farmacognosia está plenamente inserida no debate atual que permeia toda ciência e reflete tacitamente o paradigma hegemônico presente na comunidade científica da área. Porém o problema do conhecimento não se limita apenas à definição do objeto, mas diz respeito também à concepção de sujeito e de como este se relaciona com o objeto do conhecimento, como o compreende, acessa e dele se apropria. Aqui, a epistemologia implica no ensino.

Temos em vista, pela ótica da ciência, que uma das contribuições significativas da formação curricular no campo das ciências farmacêuticas corresponde à construção de uma cultura científica e tecnológica de apropriação crítica do conhecimento por parte dos nossos alunos; na medida em que, como profissionais ou mesmo como cidadãos, têm seu modo de vida, suas relações sociais, influenciados pelos resultados do que ciência e a tecnologia contemporânea produzem. Neil Postman, um crítico da excessiva submissão da sociedade moderna à tecnologia, apresentou argumentações muito apropriadas para descrever a forma como a aceitação não-crítica do conhecimento científico pode sobrepujar valores, atacar liberdades, desfigurar o patrimônio cultural e acabar por impor um tipo de dominação que o autor denomina Tecnopólio (Postman, 1994).

Não é difícil observar na tendência cada vez crescente de especialização, incorporação e valorização do aparato tecnológico para o tratamento e cura das doenças, as conseqüências dessa submissão que alimenta o estabelecimento de um paradoxo no modelo sanitário. Se por um lado os avanços científicos na área médica, na tecnologia farmacêutica e na química medicinal permitiram a descoberta e o desenvolvimento de medicamentos extremamente eficazes no combate à maioria das doenças conhecidas; de outro, o acesso a muitos desses medicamentos, principalmente nos países subdesenvolvidos, continua a ser um privilégio de apenas parcela da população (OMS, 2002). Da mesma forma, apesar deste desenvolvimento tecnológico, o processo de medicalização da sociedade parece ter mais uma contribuição negativa, no sentido do incremento dos índices de morbidade e mortalidade relacionados à farmacoterapia, do que na efetiva resolução dos problemas de saúde dos pacientes (Manasse, 1989; Otero \& Dominguez-Gil, 2000; Johnson \& Bootman, 1997).

Por sua vez, o modelo de ensino das ciências farmacêuticas, da mesma forma como ocorre com outras ciências disciplinares constantes nos currículos universitários, se não partir do pressuposto do conhecimento e da produção científica como atividade social construída, na sua determinação histórico-cultural, acaba reforçando uma transmissibilidade acrítica de conhecimentos que impede a superação do senso comum e fragiliza o posicionamento do aluno frente à compreensão do processo da medicalização, o que reproduz a submissão 
ao tecnopólio descrito por Postman (1994).

É curioso notar que frequentemente, no caso da Farmacognosia, apesar da pretendida e anunciada contextualização do ensino em torno do seu objeto do conhecimento, a saber, as plantas medicinais, percebese a influencia de concepções inatistas, do empirismo lógico e do racionalismo na gênese e abordagem do conhecimento; manifesto muitas vezes pela tendência ou persistência de ensinar um conjunto de conhecimentos que correspondem à sistematização histórica de saberes e práticas, essencialmente voltadas ao domínio de técnicas e tecnologias de desenvolvimento de substâncias biologicamente ativas e seu processamento para medicamentos, mais ou menos relacionados com recortes disciplinares específicos; respaldados nas ciências básicas e suas possibilidades de aplicação no que ora entendemos como Ciências Farmacêuticas. Tal característica reforça a idéia de evolução cumulativa e ordenada da ciência, como se esta fosse uma seara tranquila onde predominam a objetividade e neutralidade do rigor científico.

Uma simples observação da forma como o conhecimento científico é produzido cotidianamente nos laboratórios e grupos de pesquisa em Farmacognosia e Produtos Naturais, demonstra como, na prática, a gênese do conhecimento segue outra lógica.

Esta questão epistemológica, mesmo que não percebida pelos professores, implica na estruturação dos planos de ensino e na forma de ensinar a Farmacognosia. Quais conteúdos são considerados importantes? Onde fica o lugar das atividades práticas experimentais na disciplina? Elas aparecem antes ou depois da teorização? Qual o espaço da investigação ou da iniciação científica nas aulas? Para ilustrar o que isto significa, basta observar como são programadas as atividades didáticas na disciplina. Não raro, a teoria deve vir sempre antes da prática e esta quase que invariavelmente presta-se para a exemplificação de propriedades e conceitos apresentados na aula teórica.

Kuhn (2005), na sua teoria do conhecimento que permeia as comunidades científicas, descreve como a utilização de exemplares desempenha um papel no processo de ensino, de treinamento na resolução de problemas. Por outro lado aquela estrutura de aula que aparentemente reproduz o "método científico" encerra concepções inatistas do conhecimento. É importante ressaltar que as duas possibilidades guardam diferenças epistemológicas entre si, mas quase sempre nós, os educadores e pesquisadores da área, não nos damos conta disto. Recebemos nossos alunos, que trazem consigo conhecimentos prévios, concepções pré-científicas, opiniões do senso comum que podem constituir obstáculos à apropriação do conhecimento científico.

Será que estamos atentos às peculiaridades do aluno, o sujeito do conhecimento que pretendemos trabalhar nas situações organizadas de ensino na sala de aula e no laboratório?

Depois de algum tempo como docente nesta disciplina me questiono se algumas dessas perguntas não precisariam um pouco da nossa atenção!

\section{IMPLICAÇÕES PARA O ENSINO}

A introdução de uma categoria proposta por Bachelard (2002) para analisar o processo de aprendizagem e formação do pensamento científico poderá ser utilizada aqui como mais um elemento a ser considerado no ensino da Farmacognosia: a noção de obstáculo epistemológico e de ruptura entre o conhecimento do senso comum ou cultura primeira que cada aluno traz consigo para a apropriação e construção do conhecimento científico.

$\mathrm{O}$ aluno como sujeito individual, mas também coletivo, aprende constantemente, de diversas formas e em diversos cenários, inclusive fora do universo estruturado e organizado da universidade, da sala de aula, do laboratório. Quando o recebemos na disciplina este já passou por diversas situações de aprendizagem e possui além do senso comum, que corresponde às representações e percepções compartilhadas no ambiente social e cultural no qual está inserido, outro conjunto de representações, construídas dentro da universidade, resultado das interações entre sua cultura primeira e a cultura científica transmitida no ambiente acadêmico, através do treinamento, do compartilhamento de códigos, linguagens, técnicas e da generalização de conceitos que o aluno aprende tanto pela disposição em fazer parte do grupo, como por um tipo de coerção intelectual (Fleck, 1986) do grupo (representado neste caso pelo professor) sobre o aluno. Portanto este senso comum científico está engendrado a priori no processo de formação e faz parte do contexto de aprendizagem do aluno.

Alguns indícios daquele senso comum científico são percebidos no nível da linguagem e das representações dos alunos, como a confusão conceitual entre fitoterapia e homeopatia; a percepção de que o que é natural não faz mal; a percepção das substâncias bioativas como "princípios ativos"; as precipitadas conclusões sobre "verdade científica" que atribuem ingenuamente autoridade excessiva aos estudos experimentais, aos ensaios biológicos como "comprovação científica" das propriedades terapêuticas das plantas medicinais; a falta de clareza na diferenciação entre conhecimento tradicional, popular e científico e dentro deste, sobre o significado das evidências clínicas; as dificuldades na compreensão e "leitura" das estruturas químicas dos compostos bioativos etc.

Estas representações são exemplos de como o contexto simbólico e social de conhecimentos já aprendidos interagem e constituem um tipo de senso comum acadêmico do aluno, o qual poderá representar um obstáculo a ser superado para apropriação da cultura científica. Caberia ao professor, identificar os obstáculos à aprendizagem, que dificultam ou impedem a apropriação crítica de uma cultura científica. Não há um manual ou roteiro para esta tarefa, no entanto ela certamente passa pela observação e 
pela possibilidade de expressão da cultura primeira.

Uma inferência que pretendemos argumentar até aqui influencia na função do ensino da Farmacognosia na formação profissional em Farmácia. Prepara-se mais o futuro farmacêutico para compreender aspectos cognitivos e assimilar habilidades e técnicas para o exercício da Farmácia como ciência e não como prática profissional; o que constituiria mais um ponto de incongruência epistemológica no atual modelo: apesar do discurso presente na corporação que declara o farmacêutico como um profissional da saúde (o que implicitamente tenta dizer: voltado à atenção da saúde do indivíduo e da comunidade) parece contraditório com o foco tradicional de formação.

Em função do viés experimental das ciências farmacêuticas, centrado na investigação em laboratório; ou dito de outro modo, o predomínio de um modelo de racionalismo científico de base empírica, o objeto do conhecimento das ciências farmacêuticas (o medicamento), quando reproduzido na tentativa de uma prática profissional acaba sendo desastrosa, na medida em que exclui qualquer possibilidade de mediação com o indivíduo (com as pessoas que seriam as beneficiárias da prática farmacêutica) que não seja na perspectiva de considerá-lo também um objeto, quase inerte, sem autonomia, passivo.

Esta concepção, não percebida pela comunidade científica, porém assimilada pelo farmacêutico, constitui mais um obstáculo para a instauração de um novo modelo de prática profissional e constitui elemento de tensão, considerando o processo social de profissionalização onde o conjunto de conhecimentos artesanais que constituíam o corpo esotérico da Farmácia tendem a ser superados ou substituídos em função das transformações no modo de produção, do desenvolvimento tecnológico e das formas de organização na sociedade após a revolução industrial.

Não foi por acaso que a Farmacognosia foi a primeira disciplina a sofrer os impactos destas transformações, a tal ponto do seu quase desaparecimento da graduação em Farmácia em países como s EUA, sendo incorporada nos programas de Química Medicinal (Miller \& Murray, 1997). Como disciplina basilar de um modelo histórico de prática profissional ela foi uma das primeiras áreas a ver questionado seu papel na formação profissional e ainda precisa responder qual o seu lugar, sua contribuição no movimento de reorientação profissional voltado para as atividades clínicas da Farmácia.

No Brasil o desenvolvimento da Farmácia partiu do encontro entre os saberes e práticas da medicina européia do século XV e práticas curativas dos grupos étnicos habitantes do novo mundo e, apesar do estabelecimento tardio de um complexo médico-industrial que interferiu significativamente na profissão farmacêutica, esta matriz cultural permaneceu como uma característica que não deveria ser ignorada na formação e na organização curricular dos Cursos de Farmácia. Não se trata de um retorno às origens artesanais da profissão, ao tempo dos boticários, nem de trazer para o escopo da disciplina a tendência de incorporar outros conteúdos que deveriam ser contemplados em outros campos disciplinares. Há isto sim, uma necessidade, mas também a possibilidade da Farmacognosia auxiliar no estabelecimento de uma prática profissional (e de uma formação para esta prática) que realmente prepare o futuro farmacêutico como um profissional cuidador em saúde, que considere a característica da sociedade brasileira, urbana ou rural, de uma longa tradição cultural com o uso de plantas medicinais e sua assimilação e interação com outras práticas em um modelo assistencial hegemonizadado na perspectiva curativa, medicalizadora, excessivamente tecnificada, socialmente excludente e por vezes desumanizada da moderna medicina científica.

Neste processo, a seleção dos conteúdos programáticos da Farmacognosia pode contemplar esta mediação entre o científico e o conhecimento tradicional contextualizado. Qual seria o lugar, por exemplo, da inserção de espécies vegetais representantes da nossa biodiversidade nos compêndios e programas ensinados na disciplina? Como inserir e discutir com os alunos a utilização de espécies nativas de modo a conciliar a demanda dos pacientes e clientes dos serviços farmacêuticos por informação, com o estado da arte e disponibilização do conhecimento científico sobre estas espécies? São questões com implicações práticas no exercício profissional.

Novas demandas e desafios estão presentes hoje esperando por uma posição e pela contribuição disciplinar da Farmacognosia. Temas referentes às estratégias terapêuticas utilizando processos biotecnológicos, como base na biologia molecular, na recombinação de material genético, constituem uma fronteira quase ignorada nos conteúdos curriculares da Farmacognosia, pelo menos na graduação, o que não seria uma novidade já que por constituir uma região de fronteira do conhecimento novo, a tendência histórica de ensino de uma disciplina baseada nas certezas do conhecimento consolidado, encontra grande dificuldade de incorporar em sua programação a discussão da dinâmica de produção do conhecimento novo. Além disso, a incorporação de uma filosofia de cuidado como a Atenção Farmacêutica na prática profissional da Farmácia exige o desenvolvimento de competências às quais a Farmacognosia poderia contribuir (Miller \& Murray, 1997), integrando o conhecimento sobre plantas medicinais e medicamentos fitoterápicos ao contexto social, cultural e histórico que correspondem à forma como as pessoas vivem e consomem produtos para a saúde.

As recentes políticas de inserção da Fitoterapia nas ações de saúde e a regulamentação das atribuições específicas dos farmacêuticos neste cenário (Resolução No. $459,2007)$ apontam uma necessidade: partindo da óbvia constatação de que a Farmacognosia não pode sozinha, dar conta de todas as competências para que o farmacêutico possa atuar em todo o ciclo da promoção para o uso racional de plantas medicinais e seus produtos de transformação; o tema, pela sua interdisciplinaridade deveria corresponder 
a um conteúdo ou conjunto de conteúdos transversais dos projetos pedagógicos dos cursos de Farmácia. O problema é que historicamente há um entendimento velado que reserva o tema plantas medicinais quase que exclusivamente sob a responsabilidade da Farmacognosia.

Em resumo, há um dilema que precisa ser mais discutido pela comunidade de cientistas e educadores da área: os conteúdos referentes ao campo da Farmacognosia, pelo seu contexto histórico, mas também epistêmico, carregam intrinsecamente uma dimensão de conteúdo disciplinar científico básico, mas também outra dimensão de caráter técnico fundadora e potencialmente renovadora da prática profissional da Farmácia. Como conciliar estas dimensões?

Pensamos que não se trata aqui de abandonar o conjunto de conhecimentos e práticas que correspondem ao corpus desta disciplina, mas de perguntar qual a sua relação interna e externa com outros conteúdos disciplinares necessários à prática da Farmácia como profissão? Responder esta pergunta não é tarefa fácil. A realidade tem mostrado uma tendência à polarização que ou resulta no adestramento das habilidades profissionais, ou na reação das ciências básicas ou de áreas mais hard na área farmacêutica, na qual inclui-se a Farmacognosia. Não pretendemos formar profissionais sem cultura científica, pois que alienados facilmente sucumbiria a profissão sem um corpo esotérico de conhecimentos; mas também não se pode esquecer que o objetivo curricular da disciplina não para a formação em ciências farmacêuticas. Pelo menos não é este o propósito expresso nos documentos oficiais que regulam o ensino e a avaliação do ensino de Farmácia no Brasil. Apesar disso, a tentativa de unificação dessas duas dimensões expressa nas Diretrizes Curriculares Nacionais $(\mathrm{DCN})$ poderia também ser designada de "roteiro de viagem do nada para lugar nenhum", quando, depois de remendos e mais remendos, apenas expressa a visão de um profissional sem rumo, sem missão, que saberia de tudo um pouco, mas que na verdade não detém o conhecimento de quase nada.

Escolhas são necessárias em qualquer processo pedagógico. Selecionam-se conteúdos, definem-se objetivos educacionais ou de aprendizagem coerentes com a intencionalidade do desenvolvimento de competências ou de um perfil que se pretende formar. Acreditamos que se compartilhamos da concepção da determinação histórica, social e cultural do conhecimento científico; se também compreendemos e percebemos nosso aluno como um sujeito coletivo do conhecimento e atribuímos importância às interações e mediações que se processam em diferentes cenários e oportunidades de aprendizagem; se percebemos as mudanças em curso na filosofia e nos modelos de prática profissional que reorientam o foco do exercício da Farmácia para o indivíduo e; por fim, se está mais ou menos definida a resposta às primeiras perguntas formuladas no início deste texto já será um bom começo para compreender o papel e o lugar da Farmacognosia no Ensino de Farmácia e na sua reprofissionalização.

\section{REFERÊNCIAS}

Bachelard G 2002. A formação do espírito científico. $3^{\mathrm{a}}$. ed. Rio de Janeiro: Contraponto Editora. 316 p.

Decreto No. 5.813, de 22 de junho de 2006. Política Nacional de Plantas Medicinais e Fitoterápicos. Diário Oficial da União, 23 de junho de 2006.

Chang ZG, Kennedy DT, Holdford DA, Small RE 2000. Pharmacists' knowledge and attitudes toward herbal medicine. Ann Pharmacother 34: 710-715.

Dutta AP, Daftary MN, Egba PA, Kang H 2003. State of CAM education in U.S. schools of pharmacy: results of a national survey. J Am Pharm Assoc 43: 81-83.

International Pharmaceutical Federation 1997. The Tokyo Declaration (1993) Standards for quality of pharmacy services (FIP Guidelines for Good Pharmacy Practice, September 1993) and revised version FIP/WHO GPP (1997)

Fleck L 1986. La génesis y el desarrollo de un hecho científico. Madrid: Alianza Editorial.

Johnson JA., Bootman LJ 1997. Drug related morbidity and mortality and the economic impact of pharmaceutical care. Am J Health Sys Pharm 54: 554-558.

Kuhn TS. 2005. A estrutura das revoluções científicas. 9. ed. São Paulo: Perspectiva.

Manasse HR Jr. 1989. Medication use in an imperfect world: Drug misadventuring as an issue of public policy: part I. Am J Hosp Pharm 46: 924-944.

Mackowiak ED, Parikh A, Freely J 2001. Herbal product education in United States Pharmacy schools: core or elective program? Am J Pharm Educ 65: 1-6.

Miller LG, Murray WJ 1997. Herbal instruction in United States Pharmacy Schools. Am J Pharm Educ 61: 160-162.

Organización Mundial de la Salud 2002. Perspectivas Políticas sobre Medicamentos de la OMS. Promoción del uso racional de medicamentos: componentes centrales. Ginebra: OMS.

Otero MJ, Dominguez-Gil A 2000. Acontecimientos adversos por medicamentos: una patología emergente. Farm Hosp 24: 258-266.

Postman N. 1994. Tecnopólio: a rendição da cultura à tecnologia. São Paulo: Nobel.

Resolução No. 459, de 28 de fevereiro de 2007. Conselho Federal de Farmácia, Diário Oficial da União, 6 de março de 2007.

Shah B, Siganga W, Mallaya U, Shah S 2005. Pharmacy student perspectives on classroom education about herbal supplements. Am J Pharm Educ 69: Article 102.

Shields KM 2003. Natural product education in schools of pharmacy in the United States. Am J Pharm Educ 67:4348.

Tyler VE 1996. What pharmacists should know about herbal remedies. J Am Pharm Assoc NS36: 29-37 